\title{
Persistence of metabolic monitoring for psychiatry inpatients treated with second-generation antipsychotics utilizing a computer-based intervention
}

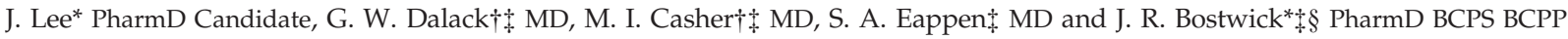 \\ *University of Michigan College of Pharmacy, Ann Arbor, MI, †University of Michigan Department of Psychiatry, Ann Arbor, MI, $\ddagger$ University of Michigan \\ Health System, Ann Arbor, MI, USA and § Present address: Room 2568B CC Little, University of Michigan College of Pharmacy, 428 Church Street, Ann \\ Arbor, MI 48109, USA
}

Received 13 October 2015, Accepted 1 February 2016

Keywords: computerized physician order entry system, metabolic monitoring, pharmacist, schizophrenia, second-generation antipsychotics

\section{SUMMARY}

What is known and objective: Monitoring and intervention for metabolic abnormalities secondary to second-generation antipsychotics (SGAs) remain weak areas of performance in mental health care. This study evaluated the sustained impact of a computerized physician order entry (CPOE) pop-up alert designed to improve rates of laboratory metabolic monitoring of patients treated with SGAs in an inpatient psychiatry unit. Interventions carried out by the psychiatry team to manage metabolic abnormalities found on screening were also identified. Methods: A retrospective chart review of patients treated with scheduled SGAs at a large Midwestern academic medical centre's inpatient adult psychiatry unit was conducted nearly 4 years after the initial implementation of a pop-up alert. Rates of laboratory monitoring (blood glucose level, haemoglobin A1C [HbA1c], lipid panel) were compared to those following the initial implementation. Medical charts of patients with abnormal laboratory results were also reviewed to summarize interventions made by the psychiatry team to manage identified abnormalities. Results and discussion: Patient demographics in the current study population $(n=129)$ were similar to those in the initial test cohort $(n=157)$. There was no significant decrease in monitoring of glucose levels and lipid panels (fasting or random). Nine patients with abnormally elevated laboratories were identified. Interventions by the psychiatry team included referrals to appropriate healthcare professionals and initiation of medication.

What is new and conclusions: The rate of metabolic monitoring for inpatients on SGA therapy did not significantly change over time with the continued use of the CPOE pop-up alert. Optimal monitoring utilizing a CPOE pop-up alert may allow the psychiatry team, including psychiatric pharmacists, to better manage metabolic conditions.

\section{WHAT IS KNOWN AND OBJECTIVE}

In response to emerging evidence supporting an association between second-generation antipsychotics (SGAs) and adverse metabolic effects, a consensus statement of guidelines was devel-

Correspondence: J. R. Bostwick, PharmD, BCPS, BCPP, University of Michigan College of Pharmacy, Ann Arbor, MI and University of Michigan Health System, Ann Arbor, MI, USA. Tel.: +1 734764 0810; fax: +1 734763 4480; e-mail: jkingsbu@med.umich.edu oped and promulgated by the American Diabetes Association, American Psychiatric Association, American Association of Clinical Endocrinologists and the North American Association for the Study of Obesity in 2004. ${ }^{1}$ The panel recommended that physicians screen and regularly monitor patients on SGAs for weight gain, the development of type II diabetes, and dyslipidemia. ${ }^{1,2}$ Moreover, the guidelines recommended that when metabolic abnormalities such as elevated fasting glucose levels were identified through monitoring, treatment should be initiated and patients referred to appropriate healthcare providers for follow-up care. ${ }^{1}$ Compliance with these recommendations has been based on two assumptions: one, that these screening measures would be implemented into psychiatrists' daily routine by virtue of greater awareness of the metabolic complications of SGAs; and two, that abnormal screening would lead to further management of these metabolic derangements, either by the psychiatrists themselves or through expedited referrals to primary care providers (PCPs). ${ }^{1}$

Despite increased awareness and general acceptance of these guidelines, appropriate metabolic monitoring has remained uncommon. In fact, several studies concluded that the publication of these guidelines in 2004 did not increase metabolic testing in patients initiating SGA therapy. ${ }^{3-5}$ One reason for this may be the uncertainty regarding the role of psychiatrists in directly managing metabolic abnormalities themselves. One study by Newcomer et $a l^{6}{ }^{6}$ found that approximately half of the psychiatrists surveyed agreed that general healthcare management was outside of their scope of practice, with nearly three-quarters of these respondents stating they would seek consultation for management of adverse metabolic effects. A 2013 study by Parameswaran et al. ${ }^{7}$ found that psychiatrists recognized the importance of screening for metabolic risk in patients receiving antipsychotic medications; but the majority of these psychiatrists believed their role was limited to metabolic screening for their patients, and that prescribing oral medications for these abnormalities remained outside of their role.

In contrast, another study by Mangurian et al. ${ }^{8}$ found that $42 \%$ of PCPs believed that psychiatrists have the responsibility to treat such abnormalities with prescription medications. These conflicting viewpoints about which healthcare providers are responsible for the treatment of metabolic abnormalities associated with SGAs pose barriers to appropriate monitoring and follow-up. Therefore, due to low rates of metabolic monitoring and presumed lack of interventions by psychiatrists (e.g. starting a statin, switching antipsychotic class, referral to internal medicine), metabolic abnormalities and cardiovascular disease are often underdiagnosed and undertreated in patients with serious mental illnesses. ${ }^{2}$ 
In response to the suboptimal metabolic monitoring of patients on SGAs, a study conducted by DelMonte et al. ${ }^{4}$ evaluated the efficacy of a computerized physician order entry (CPOE) pop-up alert designed to improve rates of laboratory metabolic monitoring of patients treated with SGAs at a large Midwestern academic medical centre's inpatient psychiatry unit. Implementation of this computerized pop-up alert led to a statistically significant improvement in the rate of ordering fasting blood glucose levels $(46.8 \%$ vs. $70 \%)$ and fasting $(18.7 \%$ vs. $59.9 \%)$ and non-fasting ( $28.7 \%$ vs. $74.5 \%$ ) lipid panels for inpatients treated with SGAs. The study did not address whether the increased rate of monitoring was sustained over time or whether abnormalities found on screening were managed by the psychiatry team.

The purpose of our follow-up study was twofold: first, to determine whether the rates of metabolic monitoring at a major academic medical centre inpatient psychiatry unit have been maintained approximately 4 years after the implementation of the CPOE pop-up alert; and second, to determine whether abnormalities found on screening were addressed by the psychiatry team.

\section{METHODS}

A retrospective chart review was conducted to compare metabolic monitoring rates for patients admitted to a large Midwestern academic medical centre's inpatient psychiatry unit between 1 January 2013 and 30 June 2013 ('current study population' most proximate to initiation of this study) to the monitoring rates for patients admitted between 3 December 2008 and 3 June 2009 after the pop-up alert was first introduced ('original post-alert study population', reported in the original DelMonte et al. study). ${ }^{4}$ The inclusion and exclusion criteria and general patient demographics for the adult inpatient psychiatry unit for this follow-up study have been previously published. ${ }^{4}$ All adult inpatients ages 18 years or older who were on a scheduled SGA during their admission were included. The term 'scheduled SGA' was defined as receiving at least two separate doses of an SGA and excluded any SGAs that were given on an 'as needed' (PRN) basis. If a patient was admitted multiple times during the study period, only the first admission was included in the study. For all study participants, patient demographics and the date and time of SGA orders, glucose levels and lipid panels were collected from the electronic medical records. This study was approved by the University of Michigan Health System Institutional Review Board.

The primary outcome of this study was the rate of metabolic monitoring, measured by the number of patients on SGAs with fasting glucose levels and/or haemoglobin A1C (HbA1c) (both referred to as 'glucose levels' for the purposes of this report), and lipid panels available. Any glucose level and lipid panel that was accessible to the inpatient team and collected from 12 weeks prior to admission to the discharge date were considered as 'baseline' laboratories available to inpatient providers for review and medical decision-making. This 12 -week window was chosen to align with monitoring recommended for SGAs in the consensus statement. ${ }^{1}$ Fasting was defined as a level drawn before 9 AM, which was the start of breakfast on the inpatient psychiatry unit and deemed reasonable by the study authors for any laboratories obtained within 12 weeks prior to admission.

This study also investigated how the psychiatry team managed metabolic abnormalities in patients identified through monitoring. From the current study population, the medical charts of patients with 'abnormal values' deemed as values of fasting LDL $>190 \mathrm{mg} /$ $\mathrm{dL}$ or triglyceride level $>500 \mathrm{mg} / \mathrm{dL}$ and non-diabetic patients with a fasting glucose level $>126 \mathrm{mg} / \mathrm{dL}$ or $\mathrm{HbA} 1 \mathrm{c} \geq 7 \%$ were reviewed to identify any interventions the psychiatry team carried out to manage these metabolic abnormalities.

To analyse the results, chi-square and student's $t$-tests were conducted using SPSS to determine whether monitoring rates and patient demographics between the current study population and original post-alert study population were significantly different. A two-sided $P$-value of $<0.05$ was considered statistically significant. The interventions made by the psychiatry team to manage metabolic abnormalities were not analysed using statistical tests, but instead reviewed and described through a case series format.

\section{RESULTS AND DISCUSSION}

\section{Patient demographics}

Comparison of demographics between the two patient populations is summarized in Table 1 . The only statistically significant differences between these groups were the prevalence of coronary artery disease/cardiovascular disease and the percentage of patients with a discharge diagnosis of substance-related disorder, both of which were significantly higher in the current study population. There were no significant differences in all other comorbidities or discharge diagnoses, age, length of stay, race, sex and SGA agents ordered between the two populations. In addition to the SGA agents listed in Table 1 (aripiprazole, clozapine, olanzapine and ziprasidone), patients were also prescribed asenapine, paliperidone, quetiapine and risperidone during their admissions.

\section{Metabolic monitoring}

Comparisons are summarized in Table 2. The number of patients with glucose levels, lipid panels and both laboratories available was not significantly different between the current study population and original post-alert study population. The same held true when comparing only fasting laboratories. There was a trend for the current study population to have a higher overall rate of monitoring fasting lipid panels. Of note, the fraction (or percentage) of all glucose levels and lipid panels ordered with or within $24 \mathrm{~h}$ of the SGA order was significantly lower for the current study population. Also, of the 91 patients with lipid panels available, the majority ( 85 patients or $93 \%$ ) had these laboratories drawn during their admission vs. in the 12 weeks prior to admission. Moreover, all 129 patients had glucose levels available, with 95\% (or 123 patients) of the laboratories drawn during admission.

\section{Interventions by psychiatry team}

Of the 129 patients treated with SGAs, nine patients had at least one fasting glucose level or fasting lipid panel value that was abnormally elevated. Specifically, three patients had a triglyceride level $>500 \mathrm{mg} / \mathrm{dL}$, one patient had a fasting $\mathrm{LDL}>190 \mathrm{mg} / \mathrm{dL}$ and five non-diabetic patients had a fasting glucose level $>126 \mathrm{mg} / \mathrm{dL}$ or $\mathrm{HbA} 1 \mathrm{c} \geq 7 \%$.

\section{DISCUSSION}

There was no statistically significant difference in metabolic monitoring rates between the current study population and the original post-alert study population. This indicates a reasonable likelihood that the overall rates of monitoring in this inpatient 
Table 1. Patient demographics of current study population compared to the original post-alert study population ${ }^{\mathrm{a}}$

\begin{tabular}{|c|c|c|c|}
\hline Patient demographics & $\begin{array}{l}\text { Current } \\
\text { study } \\
\text { population } \\
(n=129)\end{array}$ & $\begin{array}{l}\text { Original post-alert } \\
\text { study population } \\
(n=157)\end{array}$ & $P$-value \\
\hline Average age (years $\pm \mathrm{SD}^{\mathrm{b}}$ ) & $41 \pm 19$ & $42 \pm 15$ & $0 \cdot 619$ \\
\hline Length of stay (days $\pm \mathrm{SD}^{\mathrm{b}}$ ) & $16 \cdot 1 \pm 14 \cdot 3$ & $13 \cdot 4 \pm 13 \cdot 5$ & $0 \cdot 102$ \\
\hline \multicolumn{4}{|l|}{ Comorbidities } \\
\hline Diabetes & $17(13 \cdot 2)$ & $20(12 \cdot 7)$ & 0.912 \\
\hline Hyperlipidaemia & $25(19 \cdot 4)$ & $20(12 \cdot 7)$ & $0 \cdot 125$ \\
\hline $\mathrm{CAD} / \mathrm{CVD}^{\mathrm{c}}$ & $14(10.9)$ & $4(2.5)$ & 0.004 \\
\hline $\begin{array}{l}\text { BMI }>25 \text { or diagnosis } \\
\text { 'obesity' }\end{array}$ & $8(6 \cdot 2)$ & $9(5 \cdot 7)$ & $0 \cdot 867$ \\
\hline $\begin{array}{l}\text { Patients } \mathrm{w} / \geq 1 \text { of } \\
\text { the above }\end{array}$ & $46(35 \cdot 7)$ & $41(26 \cdot 1)$ & $0 \cdot 081$ \\
\hline \multicolumn{4}{|l|}{ Race } \\
\hline Caucasian & $104(80 \cdot 6)$ & $126(80 \cdot 3)$ & 0.938 \\
\hline Black/African American & $15(11 \cdot 6)$ & $16(10 \cdot 2)$ & 0.679 \\
\hline Hispanic & $0(-)$ & $3(1.9)$ & $0 \cdot 114$ \\
\hline Native American & $0(-)$ & $0(-)$ & - \\
\hline Bi/Multiracial & $0(-)$ & $1(0 \cdot 6)$ & $0 \cdot 364$ \\
\hline Asian & $3(2 \cdot 3)$ & $3(1.9)$ & 0.808 \\
\hline Middle Eastern & $0(-)$ & $2(1 \cdot 3)$ & $0 \cdot 198$ \\
\hline Unknown/Other & $3(2 \cdot 3)$ & $2(1 \cdot 3)$ & 0.500 \\
\hline Not listed & $4(3 \cdot 1)$ & $4(2 \cdot 6)$ & 0.778 \\
\hline \multicolumn{4}{|l|}{ Sex } \\
\hline Male & $70(54 \cdot 3)$ & $77(49 \cdot 0)$ & $0 \cdot 380$ \\
\hline Female & $59(45 \cdot 7)$ & $80(51 \cdot 0)$ & $0 \cdot 380$ \\
\hline \multicolumn{4}{|l|}{ Discharge diagnoses } \\
\hline $\begin{array}{l}\text { Schizophrenia/psychotic } \\
\text { disorders }\end{array}$ & $47(36 \cdot 4)$ & $44(28 \cdot 0)$ & $0 \cdot 129$ \\
\hline Depressive disorders & $34(26 \cdot 4)$ & $47(29.9)$ & 0.504 \\
\hline Bipolar disorders & $21(16 \cdot 3)$ & $39(24 \cdot 8)$ & 0.077 \\
\hline Mood disorders NOS & $10(7 \cdot 8)$ & $11(7 \cdot 0)$ & $0 \cdot 810$ \\
\hline Personality disorders & $4(3 \cdot 1)$ & $4(2 \cdot 5)$ & 0.778 \\
\hline Dementia & $1(0 \cdot 8)$ & $2(1 \cdot 3)$ & $0 \cdot 680$ \\
\hline Anxiety disorders & $6(4 \cdot 7)$ & $6(3 \cdot 8)$ & 0.728 \\
\hline Substance-related disorder & $4(3 \cdot 1)$ & $0(-)$ & 0.026 \\
\hline Adjustment disorder & $0(-)$ & $1(0 \cdot 6)$ & $0 \cdot 364$ \\
\hline Other & $2(1 \cdot 6)$ & $2(1 \cdot 3)$ & 0.843 \\
\hline \multicolumn{4}{|l|}{ SGA agent ordered } \\
\hline $\begin{array}{l}\text { Clozapine/Olanzapine } \\
\text { (high risk) }\end{array}$ & $36(27 \cdot 9)$ & $35(22 \cdot 3)$ & $0 \cdot 274$ \\
\hline $\begin{array}{l}\text { Aripiprazole/Ziprasidone } \\
\text { (low risk) }\end{array}$ & $33(25 \cdot 6)$ & $37(23 \cdot 6)$ & 0.693 \\
\hline
\end{tabular}

Data presented as $n(\%)$ unless otherwise specified.

${ }^{a}$ Original post-alert study population as reported by DelMonte et al. ${ }^{4}$ ${ }^{\mathrm{b}} \mathrm{SD}$, Standard deviation.

${ }^{\mathrm{C}} \mathrm{CAD} / \mathrm{CVD}$, Coronary artery disease/cardiovascular disease.

setting were maintained for a 4-year period, between 2009 and 2013, as the implementation of the CPOE pop-up alert. Although metabolic monitoring rates did not decrease between the two study populations over time, appropriate monitoring of glucose levels and lipid panels remained less than 100\% (optimal monitoring), as only about half of all patients who were admitted to the inpatient psychiatry unit and treated with scheduled SGAs had both fasting glucose levels and lipid panels available for monitoring during their stay. Specifically, similar to the original post-alert study population 4 years ago, $71 \%$ of patients had both a glucose level and lipid panel available, but only $51 \%$ had both laboratories drawn fasting.

Notably, the current study revealed a statistically significant decline in the number of glucose levels or lipid panels ordered with or within $24 \mathrm{~h}$ of the SGA order. This was not related to an increase in outpatient monitoring. As described in the results, the majority of patients had their laboratories drawn during their admission rather than in the 12 weeks prior to admission. Instead, this could indicate a decline in the effectiveness of the alert to trigger ordering of these laboratory parameters. The potential confounder of having a psychiatric pharmacist provides verbal reminders to prescribers (physicians or nurse practitioners) on the unit was consistent during both study periods, and this, as well as other study limitations have previously been described. ${ }^{4}$ In all, whereas the results of the study supported the long-term impact of the pop-up alert in maintaining the rates of monitoring, additional steps are still needed to increase the percentage of inpatients who have fasting glucose levels and lipid panels available while receiving SGAs. As in our previous study, the limitations inherent in our design include the retrospective non-randomized study population, the focus on only two of the multiple parameters of the metabolic syndrome, and the difficulties in securing adequate post-discharge data. However, this follow-up study was able to address the 'alert fatigue' issue that was alluded to in the previous article's discussion section.

This study also included a review of the management of identified metabolic consequences of SGAs initiated by psychiatrists and the continued care through collaboration with other healthcare providers, including nurse practitioners, psychiatry residents and a board certified psychiatric pharmacist. During the 6-month study period, only nine patients with metabolic abnormalities were identified. This small number limits our ability to generalize about the management of metabolic abnormalities by the inpatient team. Nonetheless, the medical charts of a total of nine patients with abnormal laboratory values exceeding the thresholds cited above were reviewed by the authors. Upon review, it was determined that all abnormal values were addressed appropriately (e.g. initiation of medication therapy, referral to primary care) by a psychiatry team member. All five cases of abnormal fasting glucose levels were addressed by psychiatrists and did not require any intervention. Point-of-care glucose levels continued to be monitored during admission to detect sustained elevated levels, but these patients' abnormally elevated glucose levels returned to normal and did not require interventions such as initiation of medication therapy. In addition, all three cases of elevated triglyceride levels and the one case of elevated LDL level were addressed. To provide additional texture and context to the interventions recommended, we briefly summarize three case reports illustrating the various steps taken by the psychiatry team to manage elevated fasting lipid panels identified in the current study.

Case 1: A 26-year-old transfemale with a history of bipolar I disorder and elevated triglycerides was admitted to the inpatient psychiatry unit for erratic behaviour secondary to mania and psychosis. The patient was initially treated with olanzapine as needed for severe agitation. Risperidone was then started on a scheduled basis to manage her manic symptoms and was continued for the remainder of her hospital stay. Upon discharge 1 week later, a baseline fasting lipid panel was drawn and the patient's triglyceride level was found to be $1130 \mathrm{mg} /$ $\mathrm{dL}$. The patient's discharge medication regimen included 
Table 2. Glucose and lipid monitoring rates ${ }^{\mathrm{a}}$

\begin{tabular}{|c|c|c|c|}
\hline Glucose levels & Current study population $(n=129)$ & Original post-alert study population $(n=157)$ & $P$-value \\
\hline Patients with glucose level available & $129(100)$ & $157(100)$ & - \\
\hline Subset drawn fasting & $87(67 \cdot 4)$ & $110(70 \cdot 0)$ & 0.634 \\
\hline Patients with fasting glucose level available (overall) & $87(67 \cdot 4)$ & $110(70 \cdot 0)$ & 0.634 \\
\hline Level ordered with SGA & $3(2 \cdot 3)$ & $31(19 \cdot 7)$ & $<0 \cdot 0001$ \\
\hline Subset drawn fasting & $3(2 \cdot 3)$ & $23(14 \cdot 6)$ & $<0.0001$ \\
\hline Level ordered within $24 \mathrm{~h}$ of SGA & $30(23 \cdot 3)$ & $63(40 \cdot 1)$ & 0.002 \\
\hline Subset drawn fasting & $24(18 \cdot 6)$ & $46(29 \cdot 3)$ & 0.036 \\
\hline \multicolumn{4}{|l|}{ Lipid panels } \\
\hline Patients with lipid panel available & $91(70 \cdot 5)$ & $117(74 \cdot 5)$ & $0 \cdot 452$ \\
\hline Subset drawn fasting & $81(89.0)$ & $94(81 \cdot 0)$ & 0.090 \\
\hline Patients with fasting lipid panel available (overall) & $81(62 \cdot 8)$ & $94(59.9)$ & $0 \cdot 614$ \\
\hline Panel ordered with SGA & $3(3 \cdot 3)$ & $38(32 \cdot 5)$ & $<0 \cdot 0001$ \\
\hline Subset drawn fasting & $3(3 \cdot 3)$ & $30(25 \cdot 6)$ & $<0 \cdot 0001$ \\
\hline Panel ordered within $24 \mathrm{~h}$ of SGA & $21(23 \cdot 1)$ & $57(48.7)$ & $<0 \cdot 0001$ \\
\hline Subset drawn fasting & $21(23 \cdot 1)$ & $48(41 \cdot 0)$ & 0.006 \\
\hline \multicolumn{4}{|l|}{ Both (glucose and lipid) } \\
\hline Patients with glucose level and lipid panel available & $91(70 \cdot 5)$ & $117(74 \cdot 5)$ & $0 \cdot 452$ \\
\hline Fasting glucose level and lipid panel available & $66(51 \cdot 2)$ & $75(47 \cdot 8)$ & 0.568 \\
\hline
\end{tabular}

Data presented as $n(\%)$ unless otherwise specified.

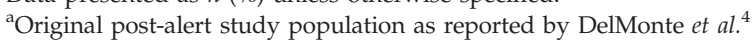

risperidone and lithium carbonate but no medications for hyperlipidemia. The patient was directed to follow up with an endocrinologist or her PCP to manage the elevated triglyceride levels. Post-discharge, as an outpatient outside of our health system, risperidone was replaced with a combination of paliperidone and bupropion for unspecified reasons. The patient also attended nutrition counselling appointments, where she received recommendations on healthy diet and lifestyle changes. Despite this education and subsequent weight loss, the patient continued to have elevated triglyceride levels. It was not until a follow-up PCP appointment approximately 5 months following her discharge from the inpatient unit that the patient was prescribed gemfibrozil and referred to a cardiology/lipid clinic. The patient was also prescribed omega- 3 fatty acids - fish oil. In reference to the elevated triglyceride levels, the PCP's documentation was that the 'timing suggests some contribution from lithium and/or paliperidone, but may have familial component as well.'

Case 2: A 29-year-old male was admitted to the inpatient psychiatry unit for an acute schizophrenia exacerbation, possibly due to medication non-adherence. During his stay, the patient was initially prescribed asenapine for acute management. This was followed by paliperidone, prior to transitioning to paliperidone palmitate long-acting injection. Initiation of the antipsychotic regimen led to symptom improvement and was well-tolerated. A lipid panel was drawn 4 days into his inpatient stay and revealed an elevated triglyceride level of $1373 \mathrm{mg} / \mathrm{dL}$ and a total cholesterol level of $364 \mathrm{mg} / \mathrm{dL}$. However, because it was drawn shortly after 9 AM, the result may not have been a true fasting level. The patient was started on simvastatin the following day and upon discharge was instructed to continue simvastatin. Two months after discharge, the patient remained on a statin, according to medical records, although it had been changed to pravastatin at an outpatient family medicine visit.
Case 3: A 29-year-old male with a history of schizophrenia was admitted to the inpatient psychiatry unit for worsening psychosis and poor oral intake. In the month prior to his admission, the patient had several medication changes. First, the patient had been switched from risperidone to olanzapine due to negative symptoms and extrapyramidal symptoms. The patient was subsequently switched to aripiprazole due to metabolic concerns. Of note, the patient had not been taking aripiprazole for at least 5 days before his admission. During his stay, the patient was started on paliperidone and transitioned to paliperidone palmitate long-acting injections. When a fasting lipid panel drawn during the hospitalization revealed an LDL level of $245 \mathrm{mg} / \mathrm{dL}$, the patient was started on simvastatin. At discharge, the patient was instructed to continue taking simvastatin and to follow up with his PCP for further management of hyperlipidemia.

These case studies illustrate the different ways inpatient psychiatrists initiated management of metabolic abnormalities that were identified through monitoring. Interventions included referrals to appropriate healthcare providers and prescribing medications, such as statins to target elevated lipids. Laboratory abnormalities were generally managed following consultation with an internist or the clinical pharmacist on the unit. With increased monitoring of metabolic parameters as supported by guidelines, the psychiatry team plays a critical role in the management of hyperglycaemia and the development of type II diabetes, dyslipidemia and obesity. If psychiatrists are able to identify and initiate treatment of metabolic conditions secondary to SGA therapy early on by active laboratory monitoring, the prevalence of metabolic complications in patients with serious mental illnesses can be reduced. This may require use of medications outside a psychiatrist's typical scope of practice or collaboration with PCPs or other healthcare providers - including clinical pharmacists - with expertise in managing these conditions. 


\section{WHAT IS NEW AND CONCLUSION}

At our institution, we have developed a system, in collaboration with an interdisciplinary team including a clinical pharmacist, to improve metabolic monitoring. With the challenges inherent in monitoring for metabolic complications in patients receiving antipsychotic medication, this study found that an inpatient CPOE pop-up alert is associated with sustained monitoring of fasting glucose levels and lipid panels for inpatients treated with scheduled SGAs. However, overall, only about half of these patients were appropriately monitored for metabolic abnormalities. Steps must be taken to ensure that all patients who are at increased for developing metabolic conditions due to SGAs be monitored during admission to inpatient psychiatry units and upon discharge. Further, it is critical that abnormal values be adequately managed when identified, either by the psychiatry team or by working in collaboration with primary care providers. Through enhanced monitoring, appropriate management, and adequate communication of abnormal findings to outpatient providers, psychiatrists and pharmacists can play a key role in the management of metabolic abnormalities.

\section{CONFLICT OF INTEREST}

The authors declare no conflict of interests.

\section{SOURCE OF FUNDING}

None.

\section{REFERENCES}

1. American Diabetes Association, American Psychiatric Association, American Association of Clinical Endocrinologists, North American Association for the Study of Obesity. Consensus development conference on antipsychotic drugs and obesity and diabetes. J Clin Psychiatry, 2004;65:267-272.

2. Vanderlip ER, Fiedorowicz JG, Haynes WG. Screening, diagnosis, and treatment of dyslipidemia among persons with persistent mental illness: a literature review. Psychiatr Serv, 2012;63:693-701.

3. Laundon W, Muzyk AJ, Gagliardi JP et al. Prevalence of baseline lipid monitoring in patients prescribed second-generation antipsychotics during their index hospitalization: a retrospective cohort study. Gen Hosp Psychiatry, 2012;34:380-384.

4. DelMonte MT, Bostwick JR, Bess JD et al. Evaluation of a computer-based intervention to enhance metabolic monitoring in psychiatry inpatients treated with second-generation antipsychotics. J Clin Pharm Ther, 2012;37:668-673.

5. Morrato EH, Druss B, Hartung DM et al. Metabolic testing rates in 3 state Medicaid programs after FDA warnings and ADA/ APA recommendations for second-generation antipsychotic drugs. Arch Gen Psychiatry, 2010;67:17-24.
6. Newcomer JW, Nasrallah HA, Loebel AD. The atypical antipsychotic therapy and metabolic issues national survey: practice patterns and knowledge of psychiatrists. J Clin Psychopharmacol, 2004;24:S1-S6.

7. Parameswaran SG, Chang C, Swenson AK. Roles in and barriers to metabolic screening for people taking antipsychotic medications: a survey of psychiatrists. Schizophr Res, 2013;143:395-396.

8. Mangurian C, Giwa F, Shumway M. Primary care providers' views on metabolic monitoring of outpatients taking antipsychotic medication. Psychiatr Serv, 2013;64: 597-599. 